\title{
Fast interaction functions for bond-based peridynamics
}

\author{
H. David Miranda ${ }^{a}$, John Orr (D) and Chris Williams ${ }^{b}$ \\ aDep. Engineering, University of Cambridge, Cambridge, UK; ${ }^{b}$ Dep. Architecture and Civil \\ Engineering, University of Bath, Bath, UK
}

\begin{abstract}
Numerical implementations of bond-based peridynamics are computationally intensive. We propose a new class of fast interaction functions for constitutive modelling that reduce calculation time when compared to other formulations in the literature. This is achieved by substituting the stretch definition from the original interaction functions with a new stretch measure that we call modified stretch. The resultant interaction functions are proven to approximate the existing formulations, and proven to require equivalent stability and convergence conditions under explicit time integration. Gains of speed greater than $11 \%$ were obtained in numerical tests that compared the new functions with those in the literature. The new approach was verified against classical elastic theory using simple examples and shows good agreement. Examples describing three-dimensional quasi-brittle structures are also presented. The proposed fast interaction functions lead to improvements in the ability to calculate the load response of realistic structures, since they usually require fine discretisation and large computation time.
\end{abstract}

\section{ARTICLE HISTORY}

Received 10 February 2018

Accepted 9 November 2018

\section{KEYWORDS}

Peridynamics; damage;

fracture; concrete

\section{Introduction}

The behaviour of certain classes of materials such as rubbers and metal alloys can be accurately predicted using the standard continuum mechanics framework (de Souza Neto, Peric, \& Owen, 2011). The equivalent predictive capability has not yet been achieved in continuum damage mechanics, despite considerable research over many years (Besson, 2010, Chaboche, 1978, Jason, Huerta, Pijaudier-Cabot, \& Ghavamian, 2006, Jing, 2003, Kachanov, 1958, Lemaitre, 1985, Murakami, 2012, Wu, Li, \& Faria, 2006, Yu, Teng, Wong, \& Dong, 2010). This research challenge arises as the partial differential equations of continuum mechanics theory cannot be used to model brittle damage without complex adaptations, resulting in procedures that do not guarantee accurate predictions in every possible scenario (Xu \& Arson, 2014). 
To avoid issues related to material continuity (when cracks open), the peridynamic theory was formulated (Silling, 2000, Silling, Epton, Weckner, $\mathrm{Xu}, \&$ Askari, 2007). Peridynamics uses an integral formulation that is valid even if disruptions of the material continuity occur, since no space derivatives are employed. Several numerical schemes to solve the equations of peridynamics have been developed (Emmrich \& Weckner, 2007, Gerstle, Sau, \& Silling, 2007, Macek \& Silling, 2007, Silling \& Askari, 2005).

One of the main disadvantages of peridynamic theory is that these numerical implementations are computationally intensive when compared to classical methods such as the finite element method (Kilic \& Madenci, 2010). The two main formulations for peridynamics found in the literature are the bondbased (Silling, 2000) and state-based (Silling et al., 2007) approaches. Statebased peridynamics is more general than bond-based peridynamics, but in some cases it is preferred since it requires significantly less computation. In this paper, we propose a novel formulation for the interaction function of the peridynamic bond-based model that is fast to compute. This is achieved by substituting the stretch definition from the original interaction functions (Silling \& Askari, 2005) with a new stretch measure that we call modified stretch. The modification is quite general in the sense that it can be applied to any interaction function defined in terms of stretch, for instance: micro linear-elastic, micro elasto-plastic, damage, and so forth.

We also show that the proposed modified stretch is a reasonable alternative for modelling damage, provided the damage occurs at relatively small strains, and that the resultant formulation requires equivalent stability conditions as the ones in the literature (Gerstle et al., 2007, Silling \& Askari, 2005) under explicit time integration schemes. The main advantage of the proposed approach is that it allows the computation of the interaction function in a large number of bonds in less time than conventional bond-based peridynamic models.

To demonstrate the potential of this alternative formulation, we apply it using the formulation of Macek and Silling (2007) within a scheme of explicit integration to the simulation of quasi-brittle structures to their rupture. Comparisons of speed, numerical verifications, and examples of applications to quasi-brittle structures are presented.

\section{Peridynamic bond-based theory}

\subsection{Fundamental equations and constitutive modelling}

In this section, a background overview of the peridynamic bond-based theory is presented. More detailed descriptions can be found in the work by Macek and Silling (2007), Silling (2000), Silling and Askari (2005). The equilibrium of a material point is described by: 


$$
\rho \ddot{\mathbf{u}}(\mathbf{x}, t)=\int_{H_{\mathbf{x}}} \mathbf{f}\left(\mathbf{u}\left(\mathbf{x}^{\prime}, t\right)-\mathbf{u}(\mathbf{x}, t), \mathbf{x}^{\prime}-\mathbf{x}\right) \mathrm{d} V_{\mathbf{x}^{\prime}}+\mathbf{b}(\mathbf{x}, t),
$$

where $t$ is an instant of time, $\mathbf{u}$ is the displacement field vector, $\mathbf{x}^{\prime}$ and $\mathbf{x}$ are the positions of the particles in the reference configuration. The interaction function $\mathbf{f}$ is a pairwise force function whose value is the force vector (per unit volume squared) that $\mathbf{x}^{\prime}$ exerts on a particle $\mathbf{x}$. The interaction between the particles $\mathbf{x}^{\prime}$ and $\mathbf{x}$ is called a bond and only occurs for pairs of particles within a certain distance called the material horizon $\delta$, corresponding to the neighbourhood $H_{\mathbf{x}}, \rho$ is the mass density in the reference configuration, and b is the prescribed body force density field.

For constitutive modelling, the interaction function $\mathbf{f}$ is expressed in terms of relative position $\xi$ and relative displacements $\mathbf{\eta}$ :

$$
\begin{gathered}
\mathbf{f}=\mathbf{f}(\mathbf{\eta}, \boldsymbol{\xi}), \\
\boldsymbol{\xi}=\mathbf{x}^{\prime}-\mathbf{x}, \\
\mathbf{\eta}=\mathbf{u}\left(\mathbf{x}^{\prime}, t\right)-\mathbf{u}(\mathbf{x}, t) .
\end{gathered}
$$

A more specific expression for the interaction function $\mathbf{f}$, given in terms of the scalar bond stretch $s$, is

$$
\mathbf{f}(\boldsymbol{\eta}, \boldsymbol{\xi})=\frac{\boldsymbol{\eta}+\boldsymbol{\xi}}{|\boldsymbol{\eta}+\boldsymbol{\xi}|} f(y(t), \boldsymbol{\xi}, t)
$$

with

$$
s=\frac{|\boldsymbol{\eta}+\boldsymbol{\xi}|-|\boldsymbol{\xi}|}{|\boldsymbol{\xi}|}=\frac{y-|\boldsymbol{\xi}|}{|\boldsymbol{\xi}|}
$$

and

$$
f(s, t, \xi)=c s .
$$

where $c$ is a stiffness material parameter. In three-dimensions, $c$ is related to the material bulk modulus $k$ by (see Macek \& Silling, 2007):

$$
c=\frac{18 \mathrm{k}}{\pi \delta^{4}}
$$

Equation (7) corresponds to a linear elastic model, although damage and plasticity models can be easily defined with a few adaptations. Following Macek and Silling (2007), the interaction function used to define damage with a brittle micro-elastic material is given by

$$
f(s, t, \xi)=\mu(t, \xi) c s,
$$




$$
\mu(t, \xi)=\left\{\begin{array}{lr}
1 & \text { if } s\left(t^{\prime}, \xi\right)<s_{0} \quad \forall 0 \leq t^{\prime} \leq t \\
0 & \text { otherwise }
\end{array}\right.
$$

with

$$
s_{0}=\sqrt{\frac{10 G_{0}}{\pi c \delta^{5}}} .
$$

where $s_{0}$ is the critical stretch for failure and $G_{0}$ is the material energy release rate. Equation (11) is valid only in three-dimensions. The damage at a certain point is defined as

$$
\varphi(\mathbf{x}, t)=\frac{1-\int_{H_{\mathbf{x}}} \mu(\mathbf{x}, t, \boldsymbol{\xi}) \mathrm{d} V_{\xi}}{\int_{H_{\mathbf{x}}} \mathrm{d} V_{\xi}} .
$$

The discretised version of the Equation (12) becomes

$$
\varphi_{k}(t)=\varphi\left(\mathbf{x}_{k}, t\right)=\frac{1-\sum_{k=1}^{N} \mu\left(\mathbf{x}_{k}, t, \boldsymbol{\xi}_{k}\right)}{N},
$$

where $N$ represents the number of bonds initially connected to a certain discretisation point $\mathbf{x}_{\boldsymbol{k}}$.

A micro-plastic material (Macek \& Silling, 2007) can be described through an interaction function defined by

$$
f(s, t, \xi)=\left\{\begin{array}{cl}
c(s-\hat{s}(t)) & \text { if }|\xi| \leq \delta \\
0 & \text { otherwise }
\end{array}\right.
$$

and

$$
\hat{s}(0)=0, \quad \dot{\hat{s}}=\left\{\begin{array}{cc}
\dot{s} & \text { if }|s-\hat{s}| \leq s_{Y} \\
0 & \text { otherwise }
\end{array},\right.
$$

where $\hat{s}(t)$ is the plastic stretch history and $s_{Y}$ the yielding stretch. The force at yielding $f_{Y}$ is

$$
f_{Y}=c s_{Y}
$$

The model described above requires only one elastic constant $c$ (Equation (8)), whereas isotropic linear elastic materials in classical theory are characterised by two such constants (Silling \& Askari, 2005). As described by Silling (2000), the bond-based model given above has a Poisson's ratio of $1 / 4$ in $3 \mathrm{D}$ problems and $1 / 3$ in $2 \mathrm{D}$ plane strain problems.

The peridynamic formulation defined in Equation (1) leads to no natural boundary conditions (Silling, 2000) and therefore surface traction and point forces cannot be applied as boundary conditions, as their integration results in zero, as shown by Kilic (2008). The method employed in the validation and numerical tests in this paper consists of applying 
forces and kinematical restraints in several layers of points close to the loaded surface or supports.

Equation (8) relates the classical parameter bulk modulus with the elastic constant of the model; however, the expression is only valid if it is assumed that the material point is within a sphere with the radius of the material horizon, $\delta$, and this condition is not satisfied near to free surfaces or interfaces with other materials. Therefore, a correction strategy for the parameter $c$ that takes into account proximity to free surfaces and interfaces provided by Macek and Silling (2007) was employed in this article. Following the work of Jason et al. (2006), we do not use volume correction for particles that are only partially within the material horizon in this paper. For a fixed number of particles, the approximation error obtained by bond-based peridynamics due to surface effects is so much larger than that due to volume effects, that such volume effects are practically negligible. Therefore, for the purposes of this paper, it was decided that the requirement for volume correction calculations would slow down the method without meaningful improvement of accuracy. Although, it may be useful to include such a correction in future work.

\subsection{Modified bond stretch}

The evaluation of the bond stretch, s, as defined in Equation (6), results in the computation of square roots, which is known to be relatively slow. To improve performance, we propose a new definition for the bond stretch that does not require evaluation of square roots. The modified bond stretch $\bar{s}$ is defined as

$$
\bar{s}=\frac{y^{2}-|\xi|^{2}}{2|\xi|^{2}}
$$

Notice that Equation (17) is simply a proposition where $\bar{s}$ converges to $s$, because both functions tend to zero when $y$ tends to $|\xi|$ (i.e. $\boldsymbol{\eta}$ tends to zero). These same considerations are also valid when computing $s$ in Equations (10) and (14). The modified bond stretch $\bar{s}$ converges to the bond stretch s, when the relative displacement field $\boldsymbol{\eta}$ converges to zero, since:

$$
\bar{s}=\frac{y^{2}-|\xi|^{2}}{2|\xi|^{2}}=\frac{(y-|\xi|)(y+|\xi|)}{2|\xi|^{2}} \approx \frac{(y-|\xi|) 2|\xi|}{2|\xi|^{2}}=\frac{y-|\xi|}{|\xi|}=s .
$$

Furthermore, both measures of stretch have the same first-order Taylor expansion:

$$
s(\mathbf{\eta}, \boldsymbol{\xi})=s(\mathbf{0}, \mathbf{0})+\frac{\partial s}{\partial \boldsymbol{\eta}}(\mathbf{0}, \boldsymbol{\xi}) \mathbf{\eta}+\mathcal{O}\left(\boldsymbol{\eta}^{2}, \boldsymbol{\xi}\right),
$$


where $s(0,0)=0$ and $\mathcal{O}\left(\eta^{2}, \xi\right)$ represent the error resulting from truncation of the terms of second and higher orders. With some calculations, the first derivatives of the bond stretch $s$ and modified bond stretch $\bar{s}$ can be obtained as follows:

$$
\begin{gathered}
\frac{\partial s}{\partial \boldsymbol{\eta}}=\frac{\partial}{\partial \boldsymbol{\eta}}\left(\frac{|\boldsymbol{\eta}+\boldsymbol{\xi}|-|\boldsymbol{\xi}|}{|\boldsymbol{\xi}|}\right)=\frac{\boldsymbol{\eta}+\boldsymbol{\xi}}{|\boldsymbol{\xi}||\boldsymbol{\eta}+\boldsymbol{\xi}|}, \\
\frac{\partial \bar{s}}{\partial \boldsymbol{\eta}}=\frac{\partial}{\partial \boldsymbol{\eta}}\left(\frac{|\boldsymbol{\eta}+\boldsymbol{\xi}|^{2}-|\boldsymbol{\xi}|^{2}}{2|\boldsymbol{\xi}|^{2}}\right)=\frac{\boldsymbol{\eta}+\boldsymbol{\xi}}{|\boldsymbol{\xi}|^{2}} .
\end{gathered}
$$

Replacing the terms from Equations (20) and (21) in Equation (19) with $\boldsymbol{\eta}=\mathbf{0}$, the same first-order Taylor expansion is obtained for both parameters $\bar{s}$ and $s$ :

$$
\bar{s}(\boldsymbol{\eta}, \xi) \approx \frac{\xi}{|\xi|^{2}} \boldsymbol{\eta} \approx s(\boldsymbol{\eta}, \xi) .
$$

Note that in Equation (22), the product between the vectors $\boldsymbol{\xi}$ and $\boldsymbol{\eta}$ is a scalar product and results in a scalar. It is shown above that, despite bond stretch $s$ and modified bond stretch $\bar{s}$ providing different forms of quantifying stretch, they converge for infinitesimal bond deformations. In this paper, we assume that the threshold stretch for damage or plastic flow of the bonds (Equation 10) is small enough to be considered infinitesimal; consequently, the use of either stretch $s$ or modified stretch $\bar{s}$ produces a similar result in the interaction function (Equation (9)). Nevertheless, large body deformations due to cracking can still be reproduced by damage of individual bonds.

\subsection{Peridynamic stretch measures under finite deformations}

In this study, large deformations due to bond stretch without damage are not employed since we assume a damage threshold stretch that is relatively small. However, for illustrative purposes, we provide a comparison and relate the stress measures under finite deformations in this section. The two measures can be compared computing the relative difference

$$
d_{r}(\mathbf{\eta}, \xi)=\frac{\bar{s}-s}{s} .
$$

Substituting Equations (6) and (17) on Equation (23) leads to

$$
d_{r}=\left(\frac{y^{2}-|\xi|^{2}}{2|\xi|^{2}}-\frac{y-|\xi|}{|\xi|}\right)\left(\frac{y-|\xi|}{|\xi|}\right)^{-1}
$$


The simplification of Equation (24) and substituting again Equation (6) results in Equation (25):

$$
d_{r}=\frac{1}{2} \frac{y-|\xi|}{|\xi|}=\frac{1}{2} s
$$

Substituting $d_{r}$ given by Equation (23) in Equation (25) and rearranging the equation results in a direct relationship between the two measures of stretch:

$$
\bar{s}=\frac{1}{2} s^{2}+s .
$$

The relation between modified stretch and stretch is represented in Figure 1.

A large stretch of $100 \%$ corresponds to $s=1$ and to a modified stretch $\bar{s}=$ 1.5 leading to a relative difference of 50\%. Therefore, from Equations (23) and (26) it can be concluded that a relatively large stretch leads to a significant relative difference and a very different modified stretch. Nevertheless, both the stretch measures mentioned above are valid for describing small deformations and may be used, depending on appropriateness for a particular problem. In the brittle micro-elastic model defined in Equation (10), the interaction function only depends on the stretch up to the critical stretch $\mathrm{s}_{0}$, after which point it is constant. The same can be said of the micro (perfectly) plastic model in Equation (15), where the interaction function only depends on the stretch up to the yielding stretch $s_{Y}$. Therefore, in the brittle micro-elastic and micro-plastic material models

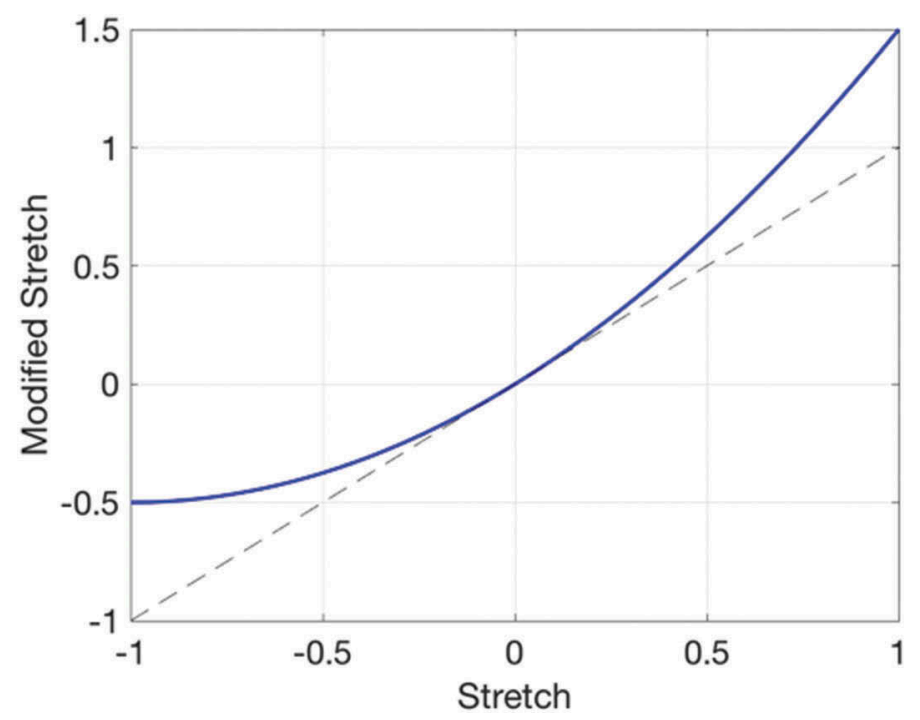

Figure 1. Relation between stretch and modified stretch under finite strains. 
presented above, the interaction function only depends on the stretch up to a certain threshold that can be considered infinitesimal, and after that the value of the interaction function remains constant. Nevertheless, other interaction functions can be employed; the only requirement is that the function assumes a constant value after a certain threshold that can be considered infinitesimal. Consequently, it is reasonable to use either stretch or modified stretch within the interaction functions.

\subsection{Stability conditions}

A discretised version of the equilibrium equation (Equation (1)) is presented by Silling and Askari (2005), and corresponds to the sum given by

$$
\rho \ddot{\mathbf{u}}_{i}^{n}=\sum_{p} \mathbf{f}\left(\mathbf{u}_{p}^{n}-\mathbf{u}_{i}^{n}, \mathbf{x}_{p}-\mathbf{x}_{i}\right) V_{p}+\mathbf{b}_{i}^{n},
$$

where $\mathbf{f}$ is given in Equation (2), $n$ is the time step number, and the subscript denotes the node number. Therefore, $\mathbf{u}_{p}^{n}$ is given as

$$
\mathbf{u}_{i}^{n}=\mathbf{u}\left(\mathbf{x}_{i}, t^{n}\right) .
$$

For a uniform rectangular grid with spacing $\Delta x$, the volume $V_{p}$ associated with the node $p$ is

$$
V_{p}=\Delta x^{3}
$$

A stability analysis of Equation (27) is described by Macek and Silling (2007), and considers the linearisation:

$$
\rho \ddot{\mathbf{u}}_{i}^{n}=\sum_{p} \mathbf{C}\left(\mathbf{x}_{p}-\mathbf{x}_{i}\right)\left(\mathbf{u}_{p}^{n}-\mathbf{u}_{i}^{n}\right) V_{p}+\mathbf{b}_{i}^{n},
$$

where $\mathbf{C}$ is a tensor-valued function called the micromodulus. In Appendix A, we show that the micromodulus is the same using the modified bond stretch $\bar{s}$ or the bond stretch $s$. Therefore, we can adopt the condition of stability from (Silling \& Askari, 2005):

$$
\Delta \mathrm{t}<\sqrt{\frac{2 \rho}{\sum_{p} V_{p}\left|\mathbf{C}\left(\mathbf{x}_{p}-\mathbf{x}_{i}\right)\right|}} .
$$

Furthermore, the spatial and temporal discretisation errors associated with Equation (30) can be shown to be $O\left(\Delta x^{2}\right)+O\left(\Delta t^{2}\right)$ as in the work by Silling and Askari (2005). 


\section{Numerical implementation}

\subsection{Initialisation of particles and bonds}

Consider a system composed of a set of $M$ material particles, and a set of $N$ bonds connecting pairs of particles. Each particle $j \in[1, \ldots, M]$ consists of:

- position $\boldsymbol{x}_{\boldsymbol{j}}$

- velocity $\boldsymbol{v}_{j}$

- resultant interaction force $f_{j}$

- externally applied force $\boldsymbol{f}_{j}^{\text {ext }}$

- boundary condition $\boldsymbol{c}_{j}$

- mass $m_{j}$

Each bond $i \in[1, \ldots, N]$ connects two particles 1 and 2, and consists of:

- logic flag indicating if the bond is broken, $\mu_{i}$

- the number of particles that connect to 1 and 2

- reference of the material connecting the particles

All particles are initialised and distributed according to a regularly spaced rectangular lattice. The connection bonds are then initialised for all pairs of particles within an initially specified material horizon $\delta_{\text {horiz }}$.

\subsection{Explicit integration scheme}

Following Gerstle (2015), dynamic relaxation (Day, 1965, Otter, 1965) was employed to improve model convergence by introducing kinematic damping. An Euler explicit integration scheme was used, following the algorithm given below:

(0) $\psi=\left(1+\frac{\Delta t}{\rho} c_{v}\right)^{-1}$

(1) loop

(2) $t=t+\Delta t$

(3) $\quad F=\min \left(t / t_{\max } ; 1.0\right)$

(4) forall bonds $i$ (connecting particles 1 and 2 )

(5) update the stretch : $s=s\left(\boldsymbol{x}_{1}, \boldsymbol{x}_{2}\right)$

(6) update broken bond flag: $\mu_{i}=\mu(s)$

(7) compute interaction force: $\boldsymbol{f}_{i}=\boldsymbol{f}\left(\boldsymbol{x}_{1}, \boldsymbol{x}_{2}, s, \mu_{i}\right)$

(8) sum interaction force to the particles: $\boldsymbol{f}_{1}=\boldsymbol{f}_{1}+\boldsymbol{f}_{i} \boldsymbol{f}_{2}=\boldsymbol{f}_{2}-\boldsymbol{f}_{i}$

(9) endfor

(10) forall particles $j$

sum the external loads to the particles: $\boldsymbol{f}_{j}=\boldsymbol{f}_{j}+F \boldsymbol{f}_{j}^{\text {ext }}$ 
(20) endfor

update velocity: $\boldsymbol{v}_{j}=\psi\left[\boldsymbol{v}_{j}+\left(\Delta t / m_{j}\right) \boldsymbol{f}_{j}\right]$ if $j$ is constrained then set position to match the constrain: $\boldsymbol{x}_{j}=F \boldsymbol{c}_{\boldsymbol{i}}$ set velocity to match the constrain: $\boldsymbol{v}_{\boldsymbol{j}}=\boldsymbol{c}_{\boldsymbol{i}} \Delta t / t_{\text {max }}$ else endif update position: $\boldsymbol{x}_{j}=\boldsymbol{x}_{j}+\boldsymbol{v}_{j} . \Delta t$

(21) endloop

The factor $\psi$, which was used to incorporate dynamic relaxation (Day, 1965, Otter, 1965), is computed considering the time step $\Delta t$, the density $\rho$, and the damping coefficient $c_{v}$, with:

$$
\psi=\left(1+\frac{\Delta t}{\rho} c_{v}\right)^{-1} \text { and } 0>\psi \leq 1
$$

The loading factor $F$ represents a ratio between the loads or displacements applied at a given instant of time and their maximum, and is given by

$$
F=\min \left(t / t_{\text {max }} ; 1.0\right) \quad \mid 0 \leq t \leq t_{\text {total }}
$$

where $t_{\text {max }}$ indicates the time where the actions are applied with maximum intensity and $t_{\text {total }}$ is the total time of the simulation $\left(t_{\text {max }} \leq t_{\text {total }}\right)$.

The update of the broken bond flag $\mu_{\mathrm{i}}$ is given in Equation (17). Therefore, if the threshold stretch $s_{0}$ from Equation (11) is achieved, the bond breaks. The computation of the interaction force is made according to Equation (9) considering $\boldsymbol{\xi}=\boldsymbol{x}_{2}-\boldsymbol{x}_{1}$, and the constant $c$ determined according to Equation (8). The algorithm was implemented in the $\mathrm{C}$ programing language and for post processing of the results the software Paraview (Squillacote \& Ahrens, 2007) and Matlab (Higham \& Higham, 2005) were employed.

\subsection{Relation between particles, bonds, and material horizon}

The number of particles $M$ and number of bonds $N$ are important to determine the computation time. As a consequence of the initiation process, $N$ depends not only on the number of particles $M$ but also on the particular geometry of the body. Nevertheless, an approximated relation between $N$ and $M$ can be obtained. If the body is assumed large enough to be considered infinite, then the number of particles $M_{V}$ in a certain representative volume is 
Table 1. Approximate number of bonds per particle $(N / M)$ as a function of the material horizon $\left(\delta_{\text {horiz }} / \lambda\right)$.

\begin{tabular}{lcccccccc}
\hline$\delta_{\text {horiz }} / \lambda$ & 1 & 2 & 3 & 4 & 5 & 6 & 7 & 8 \\
\hline$N / M$ & 1.6 & 16.3 & 56.0 & 134 & 261 & 452 & 718 & 1070 \\
\hline
\end{tabular}

$$
M_{V}=V / \lambda^{3} .
$$

where $\lambda$ is the interparticle distance. The volume of material $V_{\text {horiz }}$ within the material horizon of a generic particle is

$$
V_{\text {horiz }}=4 / 3 \pi \delta_{\text {horiz }}^{3} \text {. }
$$

The number of particles $M_{\text {horiz }}$ within the material horizon is obtained substituting the volume in Equation (34) by $V_{\text {horiz }}$ from Equation (35), resulting in

$$
M_{\text {horiz }}=V_{\text {horiz }} / \lambda^{3}=4 / 3 \pi\left(\delta_{\text {horiz }} / \lambda\right)^{3} .
$$

Finally, the approximate number of bonds $N$ can be obtained computing the number of combinations of pairs of particles within the material horizon and multiplying by the number of particles:

$$
N \approx\left(\begin{array}{c}
N_{\text {horiz }} \\
2
\end{array}\right) M=\left(\left(4 / 6 \pi\left(\delta_{\text {horiz }} / \lambda\right)^{3}-1 / 2\right) M .\right.
$$

For illustration, the approximate number of bonds per particle as a function of the material horizon, computed with Equation (37), is given in Table 1.

From Equation (37) it can be concluded that while the number of bonds grows linearly with the number of particles, it grows very rapidly with the cube of the material horizon. Since the computation time depends on the number of bonds, the computation time will grow very rapidly with the material horizon.

\section{Computational time required using stretch and modified stretch}

This section presents a benchmark test where the time to compute a simple structure is evaluated using two interaction functions based on different stretch measures. These previously described definitions were the stretch Equation (6) and modified stretch Equation (17). The structure considered is shown in Figure 2. The fixed end beam is subjected to a tensile horizontal displacement of $1 \mathrm{~mm}$ (this geometry is based on Miranda, Williams, and Orr (2016)).

The computational model consists of a rectangular, uniformly spaced lattice of calculation points with interparticle spacing $\lambda$. The material horizon considered for all the calculations in this paper was $\delta_{\text {horiz }}=$ 


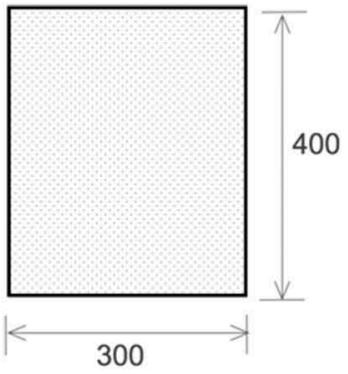

a)

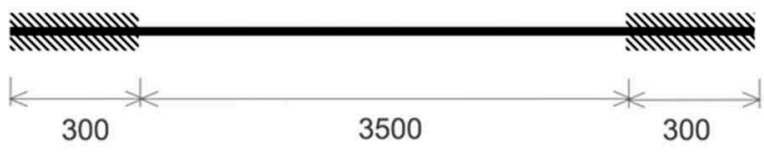

b)

Figure 2. Geometry and boundary conditions of the beam used for the benchmark. (a) cross section; (b) boundary conditions (all dimensions in millimetres).

$2 \sqrt{3} \lambda$ (i.e. three times the diagonal of a cube with edge length equal to the interparticle distance $\lambda$ ), which is in agreement with common values used in the literature (see, for instance, Hu, Ha, \& Bobaru, 2011, Le \& Bobaru, 2018, Silling \& Askari, 2005). This value was found to lead to a good tradeoff between computational effort and representation of interactions between neighbouring particles.

Simulations were performed, varying the interparticle spacing $\lambda$ and the definition of stretch. Two models were employed: model A and model B. Model A, represented in Figure 3, had an interparticle spacing $\lambda=50 \mathrm{~mm}$. Model B is more refined, with $\lambda=25 \mathrm{~mm}$. Both models were simulated considering 37,500 time increments (corresponding to a time interval of $15 \mathrm{~s}$ with increments of $4 \mathrm{e}-4 \mathrm{~s})$. Similar reaction forces and displacement fields were obtained (less than $0.1 \%$ of difference). Table 2 compares the computational time required using the definition stretch $s$ from Equation (6) and the modified stretch $\bar{s}$ from Equation (17) for each model.

The results in Table 2 confirm that Model B requires more computation time compared to Model A, as expected, since it has more particles and bonds. Nevertheless, for both models the modified stretch $\bar{s}$ led to savings in computation time: $12 \%$ for Model A and $11 \%$ for Model B.

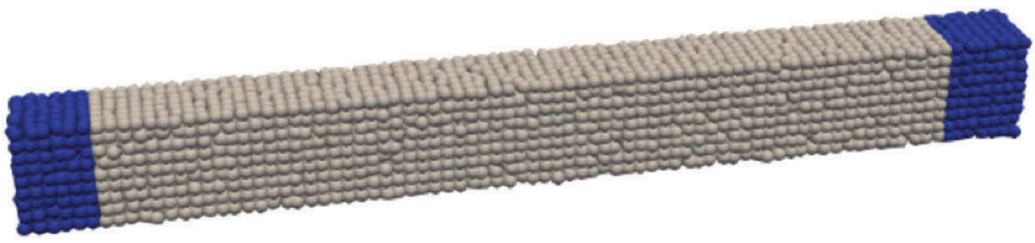

Figure 3. Representation of Model A used for the benchmark, the coloured nodes in the borders are restrained (5229 nodes, 313,710 bonds). 
Table 2. Comparison of computation times using different expressions for the stretch.

\begin{tabular}{lcc}
\hline Model & $\mathrm{A}$ & $\mathrm{B}$ \\
\hline Number of particles & 5229 & 36,465 \\
Number of bonds & 313,710 & $2,627,528$ \\
Computation time using stretch s (Equation (6)) [hh:mm:ss] & $00: 10: 39$ & $01: 30: 46$ \\
Computation time using modified stretch $\bar{s}$ (Equation (17)) [hh:mm:ss] & $00: 09: 30$ & $01: 21: 41$ \\
Relative time difference & $\mathbf{1 2 \%}$ & $\mathbf{1 1 \%}$ \\
\hline
\end{tabular}

Recalling the algorithm in Section 3.1.2, it was determined by measurement that around $98 \%$ of the calculation time is spent on the loop over the bonds (lines 4 to 9 ), and only $2 \%$ on the loop over the particles (lines 10 to 20). It was shown in Section 3 that the number of bonds increases with the cube of the material horizon; therefore, the computation time will also grow in the same order of the cube of the material horizon. Unfortunately, it was technically difficult to measure with acceptable precision the time of the operations within the bond loop, due to the uncertainty introduced by the measuring process in very small leaps of time. However, the savings seem reasonable provided that the computation time is shared equally with the other three operations within the dominant loop.

All the simulations in this work were performed using a single processor core in a laptop computer with a $2.5 \mathrm{GHz}$ Intel Core i7 processor, $4 \mathrm{MB}$ of cache memory, and 16 GB of dynamic RAM. From the results of Table 2, it was verified that the use of modified stretch $\bar{s}$ resulted in a reduction of the computation time above $10 \%$. However, these results may vary with the computer and architecture used to run simulations.

It can be concluded that the adaptation of the fast interaction function is very simple to implement, since it only requires substituting the stretch $s$ by modified stretch $\bar{s}$ but corresponds to an improvement in the computation speed. This is especially important in realistic problems requiring fine discretisation and large amount of computation time and resources.

\section{Numerical examples}

In this section, we show both numerical examples to validate the model under linear elasticity and examples to reproduce laboratorial experiments with quasi-brittle materials. The material horizon considered for all the calculations was $\delta_{\text {horiz }}=2 \sqrt{3} \lambda$ as explained in the previous section.

\subsection{Validations in the linear elastic regime}

To verify the implementation of the algorithm proposed in Section 3, example structures were analysed using the proposed algorithm and 
compared to the Finite Element Method and the classical analytical expressions for the deflection of beams based on the Euler-Bernoulli theory (see, for instance, Gere \& Goodno, 2012).

\subsubsection{Example 1: linear elements}

Two beams with rectangular cross sections were considered: a clamped beam and a cantilever beam (based on Miranda et al., 2016). The geometry, applied loads, and boundary conditions for the beams are given in Figure 4. The uniformly distributed load considered was $p=10 \mathrm{kN} / \mathrm{m}$. The material parameters are given in Table 3 .

where $\rho_{0}$ is the real density of the material and $\rho$ is a scaled value of the density by a factor of 100 as a strategy of dynamic relaxation to improve convergence (Day, 1965, Otter, 1965). The peridynamic models for these beams consist of three-dimensional grids of points spaced by $\lambda=0.05 \mathrm{~m}$ in each direction. The material parameter $c$ was defined from the material properties according to Equation (8) and the critical time step for simulation according to Equation (31). Kinematic damping was employed as mentioned in Section 3.1.2. The load was applied gradually and proportionally to the time over 10 initial seconds up to its maximum value and was then sustained for the next 5 seconds to allow the transient response to be damped as described by the loading curve in Figure 5 .

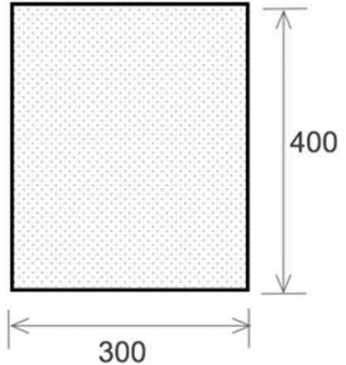

a)

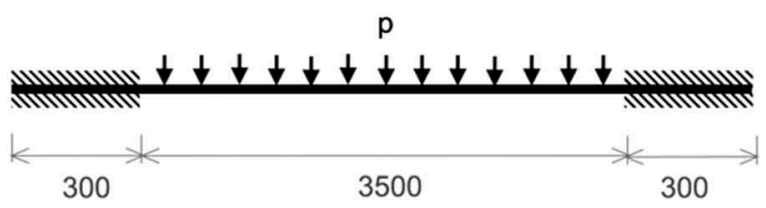

b)

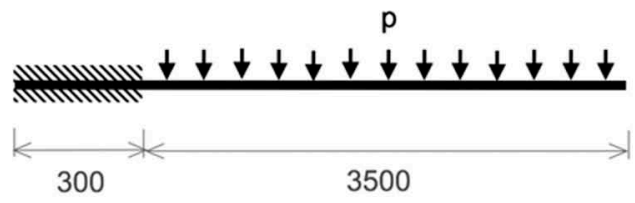

c)

Figure 4. Geometry and boundary conditions for Example 1: (a) beam cross section; (b) clamped beam; (c) cantilever beam (all dimensions in millimetres).

Table 3. Material parameters.

\begin{tabular}{lccc}
\hline$E$ & $u$ & $\rho_{0}$ & $\rho$ \\
\hline $20 \mathrm{GPa}$ & 0.25 & $25 \mathrm{kN} / \mathrm{m}^{3}$ & $2500 \mathrm{kN} / \mathrm{m}^{3}$ \\
\hline
\end{tabular}




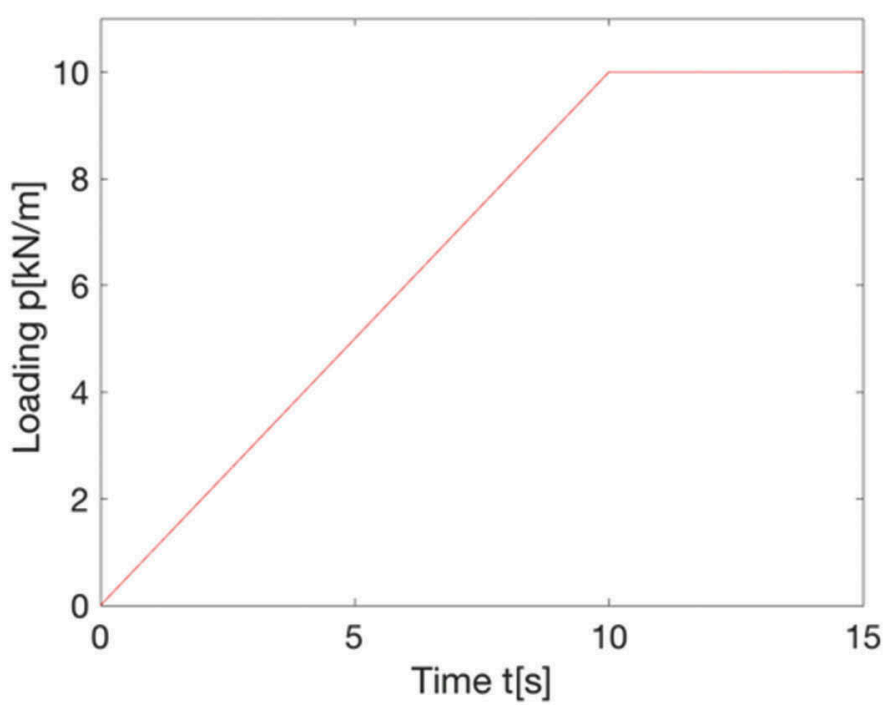

Figure 5. Loading curve.

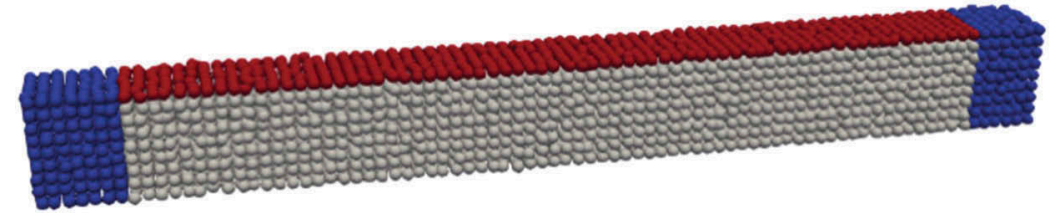

Figure 6. Model of the clamped beam (5229 particles and 313,710 bonds). Loaded particles are represented at the top layer in red and boundary conditions at the sides in blue.

Figure 6 shows the model of the clamped beam. A contour map of vertical displacements is shown in Figure 7. Figure 8 compares the deflections obtained using the analytical Euler-Bernoulli formulation against the proposed algorithm. The maximum relative difference was approximately $3 \%$.

The results for the Cantilever beam are shown in Figure 9. A comparison between the deflections using the analytical formulation and the current method is presented in Figure 10; the maximum relative difference was around $1 \%$.

Each of the examples above required less than four minutes to run (with a time step of 1e-3s corresponding to 15,000 increments) using the computer described in Section 4.

\subsubsection{Example 2: rectangular plate with three edges built in}

The rectangular plate constrained on three of its edges and subjected to a uniform load of $10 \mathrm{kN} / \mathrm{m}^{2}$ was modelled. Figure 11 describes the 


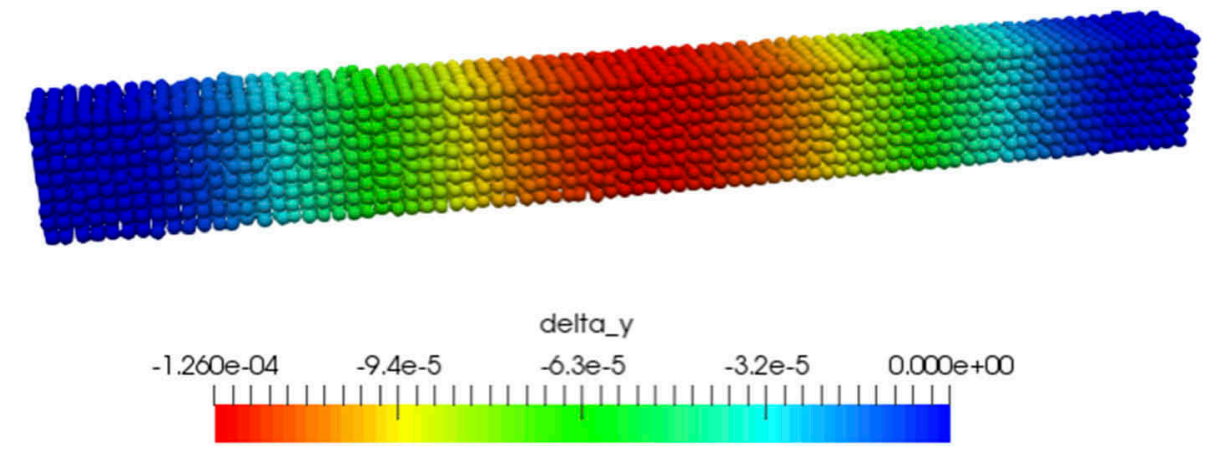

Figure 7. Deflection in meters of the clamped beam.

(5229 particles and 313,710 bonds).

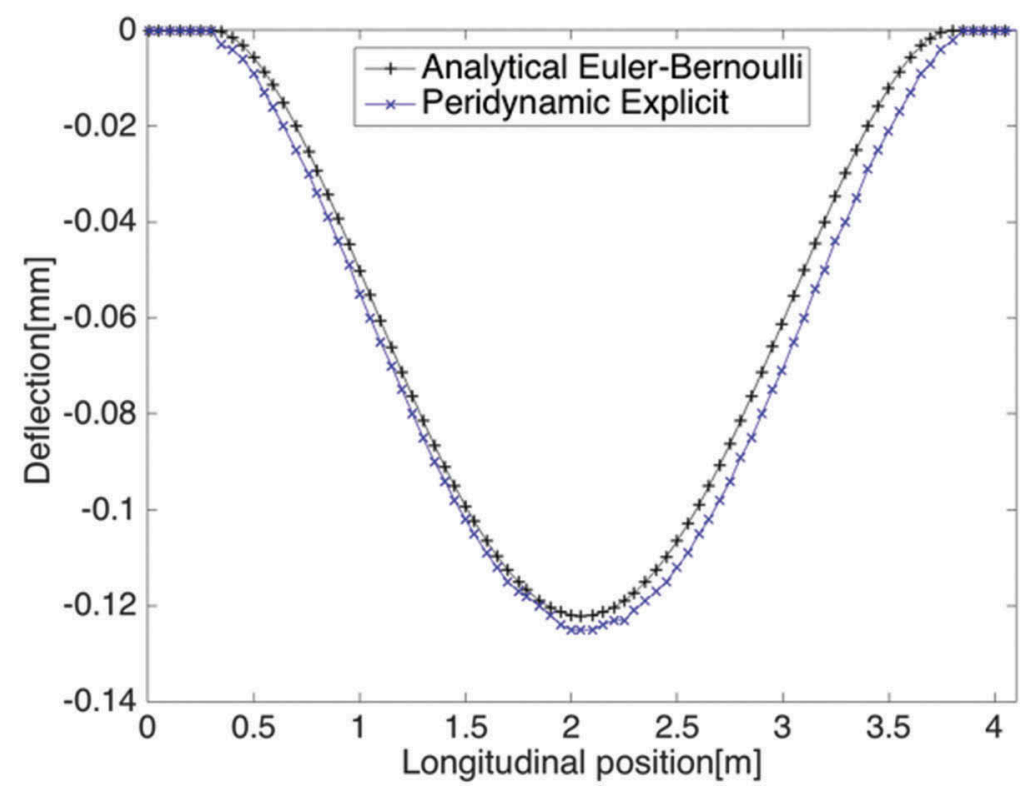

Figure 8. Comparison of deflections of the clamped beam using the analytical Euler-Bernoulli formulation and the presented method.

geometry (based on Miranda et al., 2016), boundary conditions, and loading while the material parameters are the same as in the previous example as given in Table 3.

The peridynamic model for the plate consists of a three-dimensional grid of points spaced by $\lambda=0.25 / 4 \mathrm{~m}$ in each direction. The material parameter $c$ was defined from the material properties according to Equation (8) and the critical time step for simulation according to Equation (31). The kinematic damping was employed as mentioned in Section 3.1.2. The load was applied gradually as described in the loading 

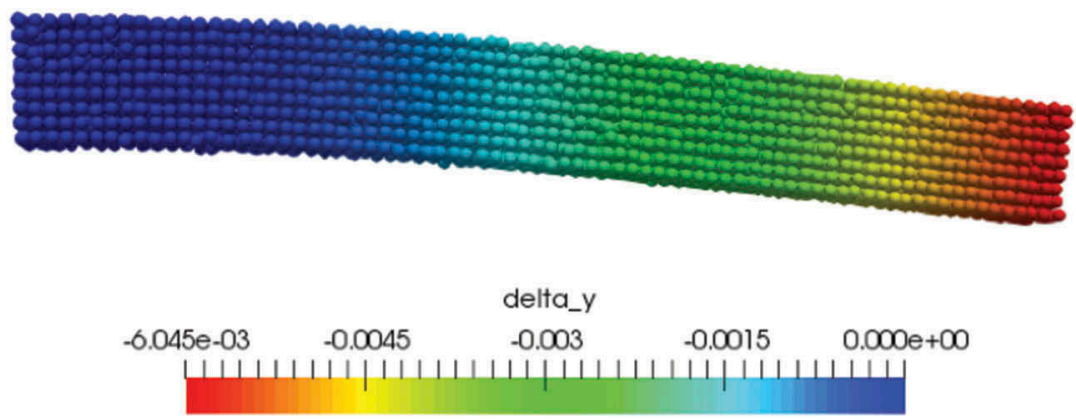

Figure 9. Deflection in meters of the Cantilever beam (4851 particles and 290,934 bonds).

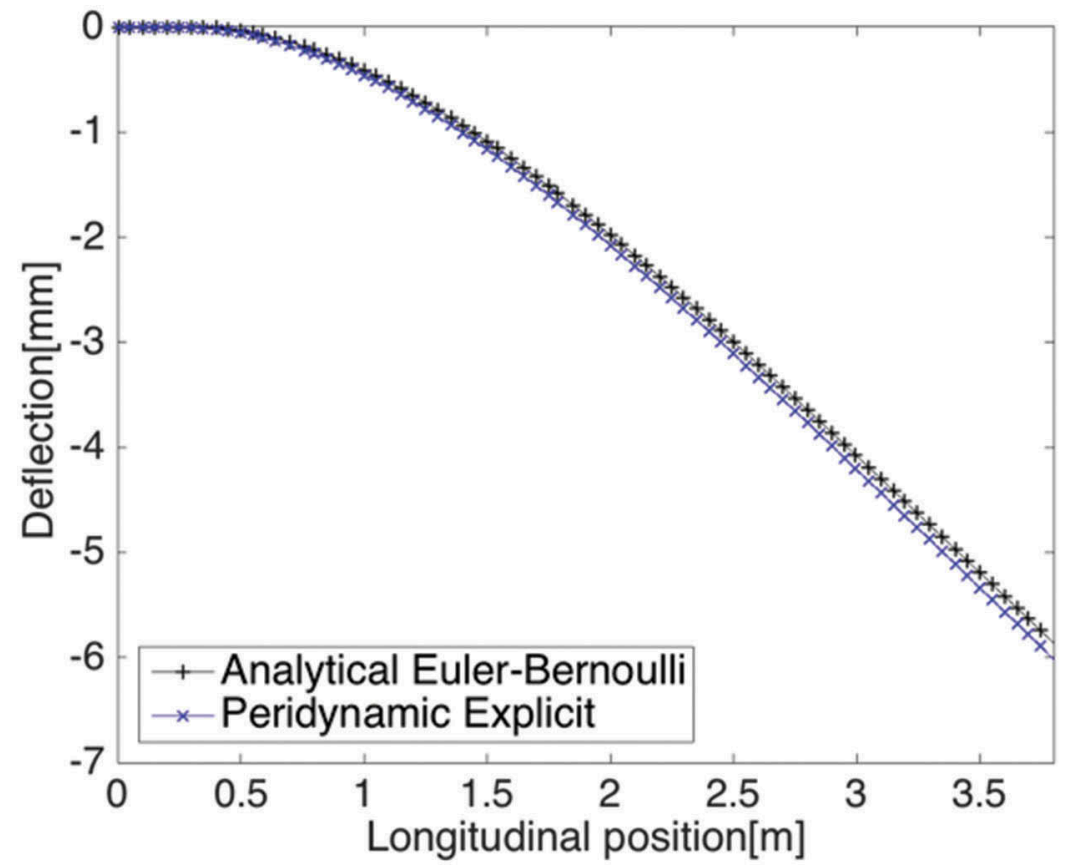

Figure 10. Comparison of displacements for the Cantilever beam.

curve from Figure 12. The time between 10 and $30 \mathrm{~s}$ is used to damp the dynamic response of the structure.

Analysis was undertaken using the finite element method and the proposed peridynamic method. The results, given in terms of deflections, are compared in Figure 13, Figure 14, and Figure 15. Figure 13 presents the deflection contour plots for both methods. Figure 14 shows the deflection curves through the centre of the slab in the perpendicular direction to the free edge and Figure 15 shows the deflection curves along the free edge.

The maximum deviation between predictions is around $4 \%$. The computational time required for the explicit peridynamic analysis was less than 


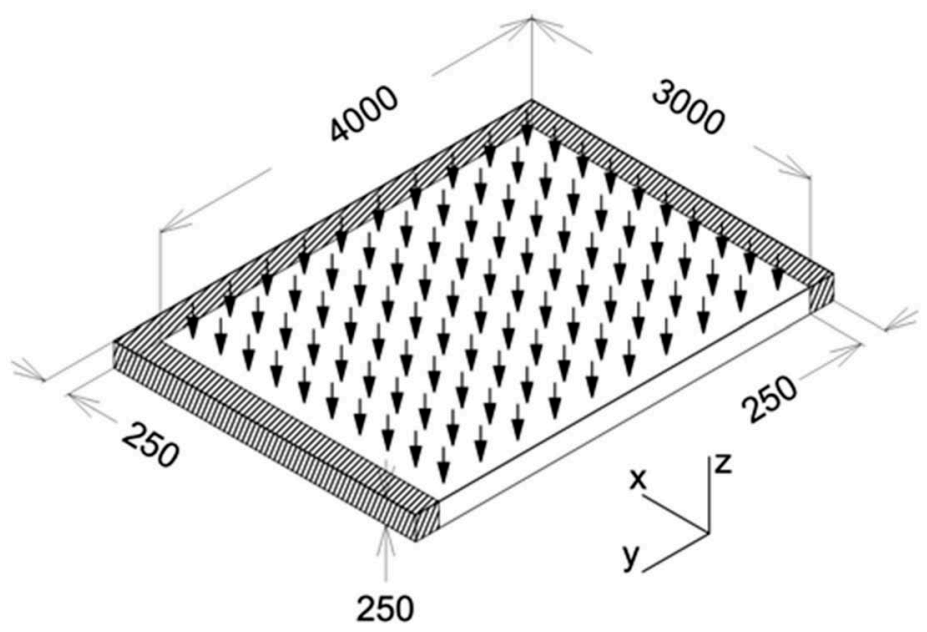

Figure 11. Model of the rectangular plate, the hatched zones are completely constrained (all dimensions in millimetres).

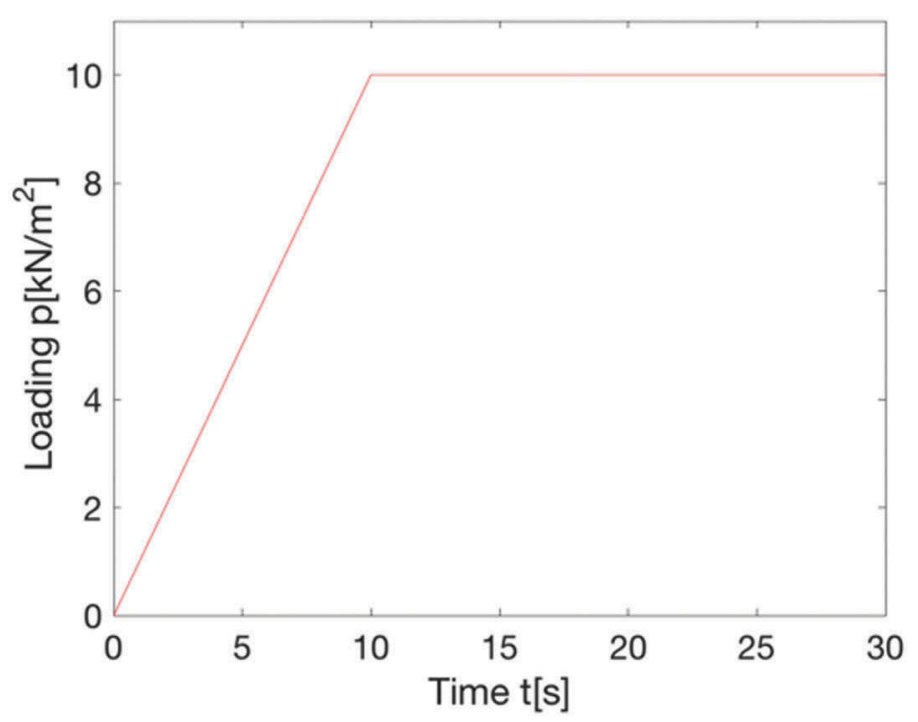

Figure 12. Loading curve.

2 min (with a time step of 15e-3s corresponding to 2000 increments) using the computer described in Section 4.

\subsection{Examples using quasi-fragile materials}

In this section, the capacity of the model to reproduce cracking patterns was explored. For simplicity and to enable comparison with real test data, 
a)
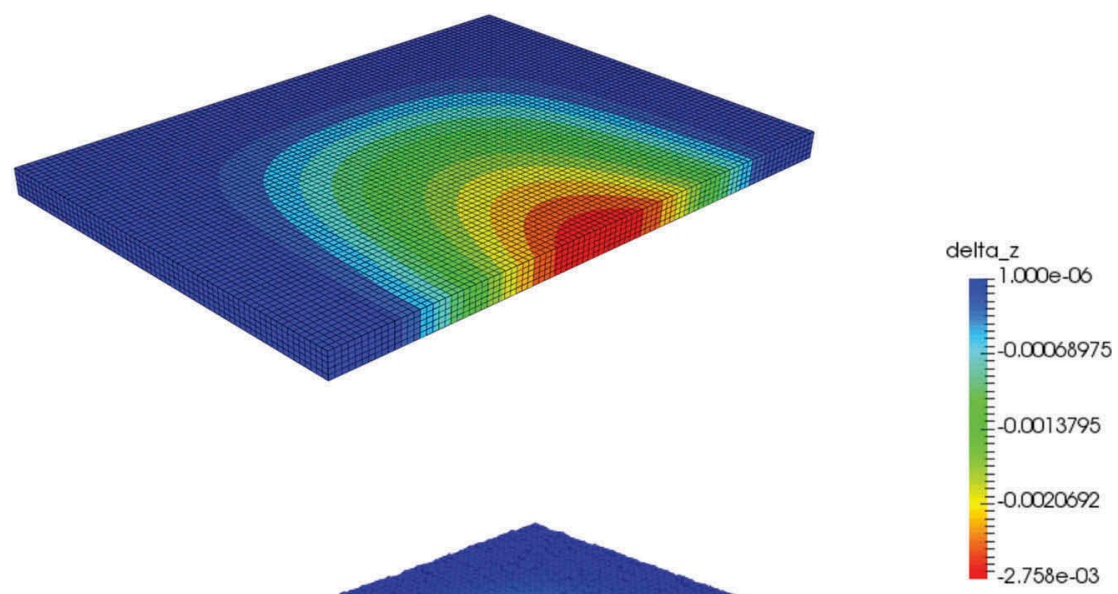

b)

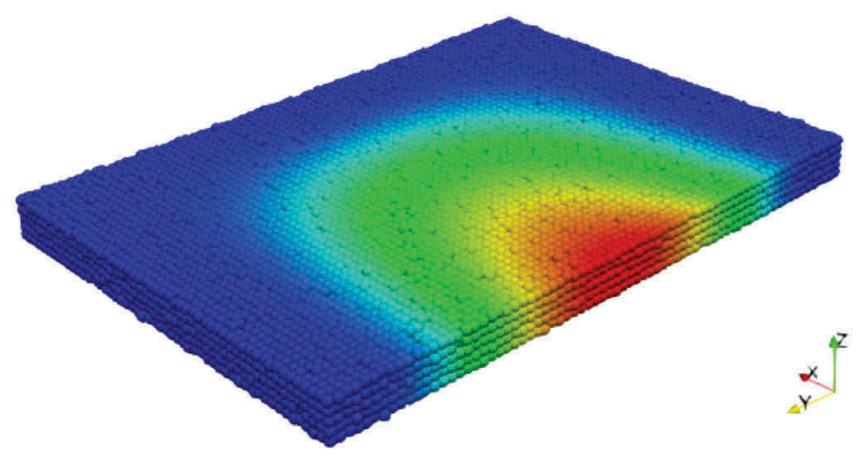

Figure 13. Contour plot of the deflection obtained using: (a) FEM (Hibbett et al., 1998) and (b) the proposed method with 19,345 particles and 1,204,382 bonds (deflection values in meters).

well-known laboratorial tests were chosen: the bending of a simply supported beam and the compression of a cube for determination of the compression strength. The examples given in this section are used to illustrate applications of the theory. A rigorous validation by laboratorial tests will form the basis of future work.

\subsubsection{Simply supported beam}

A simply supported beam (Figure 16) subject to a vertical displacement in the mid-span $\delta_{\max }=20 \mathrm{~mm}$ was modelled.

The cross section is composed of a bulk material with a similar modulus of elasticity to concrete of grade C16/20 (CEN, 1992) and reinforced with bars of a linear elastic material to model steel in its elastic range. At the interface between steel and concrete, the approach of Agwai, Guven, and Madenci (2011) was adopted. Perfect bonding is assumed, and it is postulated that rupture is initiated within the concrete, since this is a weaker material. Further work is required to study the interface between materials in more detail. Therefore, the interface connections between particles of the steel reinforcement and particles of concrete are assumed to have the same properties as the 


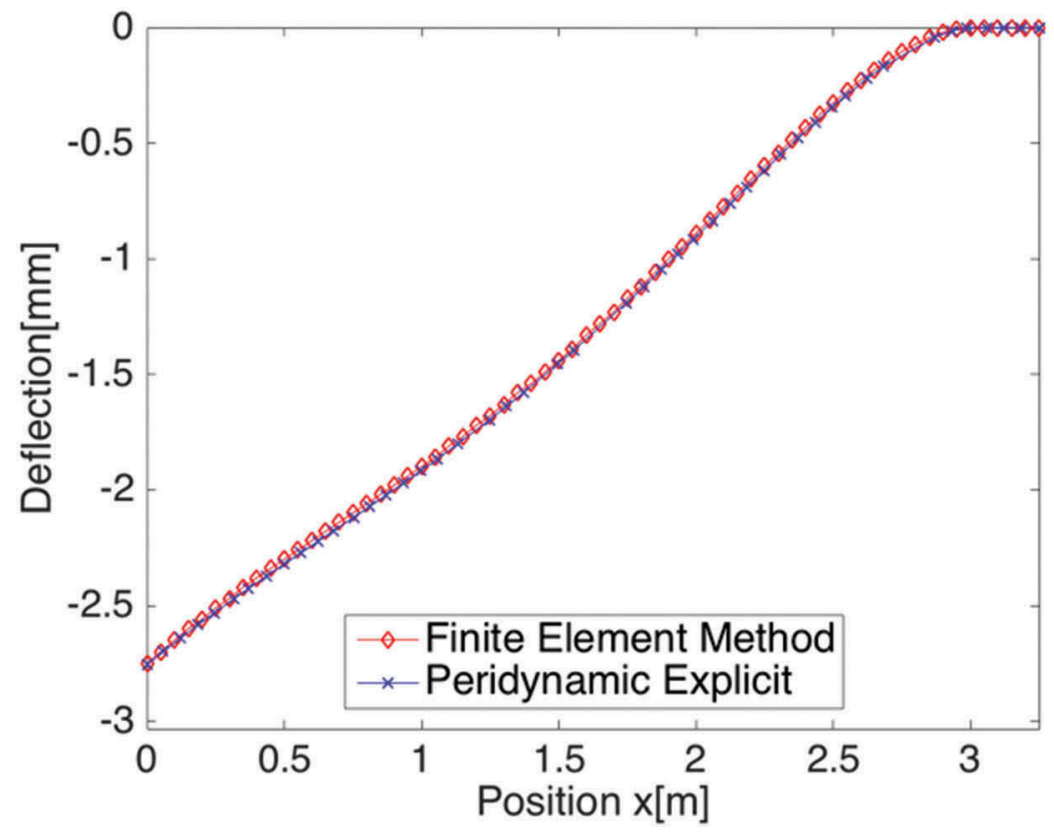

Figure 14. Plate deflection along the central line perpendicular to the free edge ( $x$-axis).

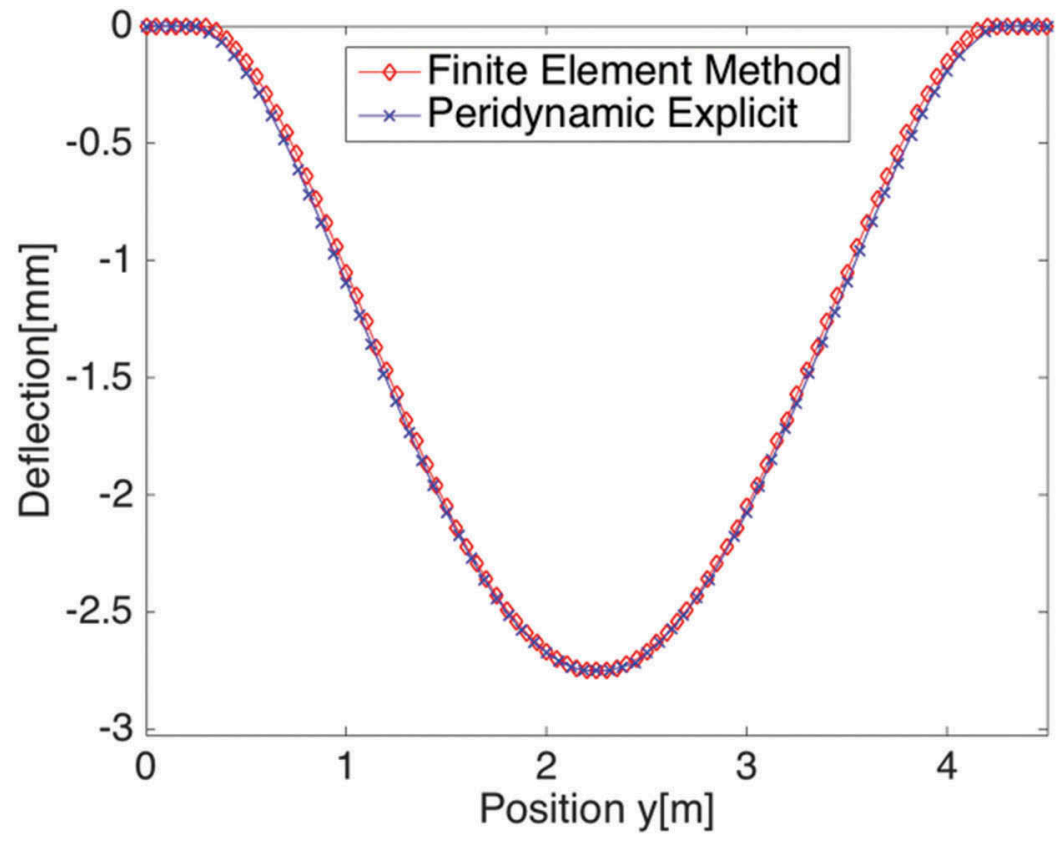

Figure 15. Plate deflection along the free edge (y-axis). 


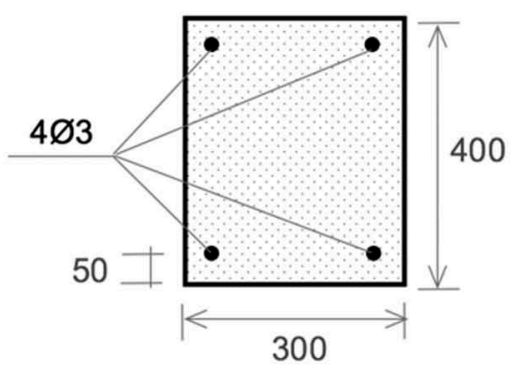

a)

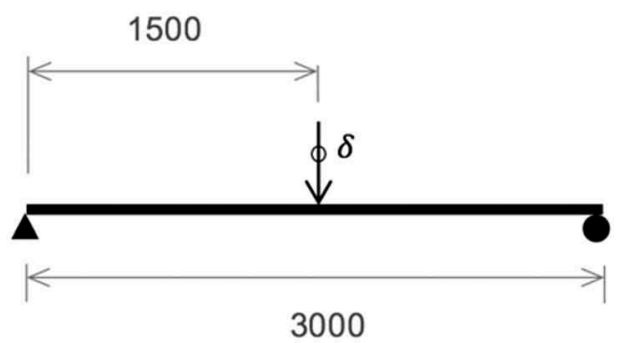

b)

Figure 16. Geometry and boundary conditions of a simply supported beam: (a) reinforced cross section; (b) support conditions and imposed displacement (all dimensions in millimetres).

Table 4. Parameters of the materials.

\begin{tabular}{lccrcc}
\hline Bulk material & & & & \multicolumn{2}{c}{ Reinforcement } \\
\cline { 5 - 6 }$E_{c}$ & $u_{c}$ & Tensile strength $f_{c t}$ & & $E_{s}$ & $U_{s}$ \\
\hline $20 \mathrm{GPa}$ & 0.25 & $2.0 \mathrm{MPa}$ & & $200 \mathrm{GPa}$ & 0.25 \\
\hline
\end{tabular}

connections between concrete particles. The properties of the cross-section materials are indicated in Table 4.

The beam model was discretised with 13.5 thousand particles and 2.71 million bonds connecting these particles. A scalar variable $\mathrm{D}$, describing the level of damage of a particle, was considered, as defined in Equation (13) which leads to

$$
\mathrm{D}=N_{d} / N_{p},
$$

where $N_{d}$ is the number of broken bonds and $N_{p}$ is the total number of bonds initially connected to the particle.

Figure 17 shows the damage levels obtained at $37 \%$ of the maximum displacement. It was found that damage by spalling develops in the region where the displacement is applied and near the supports due to the concentrated stress developed in the absence of distributing reinforcement, as is apparent in Figure 18. Failure occurs in shear due to the lack of vertical elements of reinforcement in the model, as shown in Figure 18 and Figure 19. Figure 20, from a similar laboratory experiment, shows the same typical mode of failure as the one obtained in the numerical experiment.

\subsubsection{Simulation of the standard testing of concrete compressive strength}

The normalised method for determining the compressive strength of concrete (Scrivener, Crumbie, \& Laugesen, 2004) was simulated. In this 


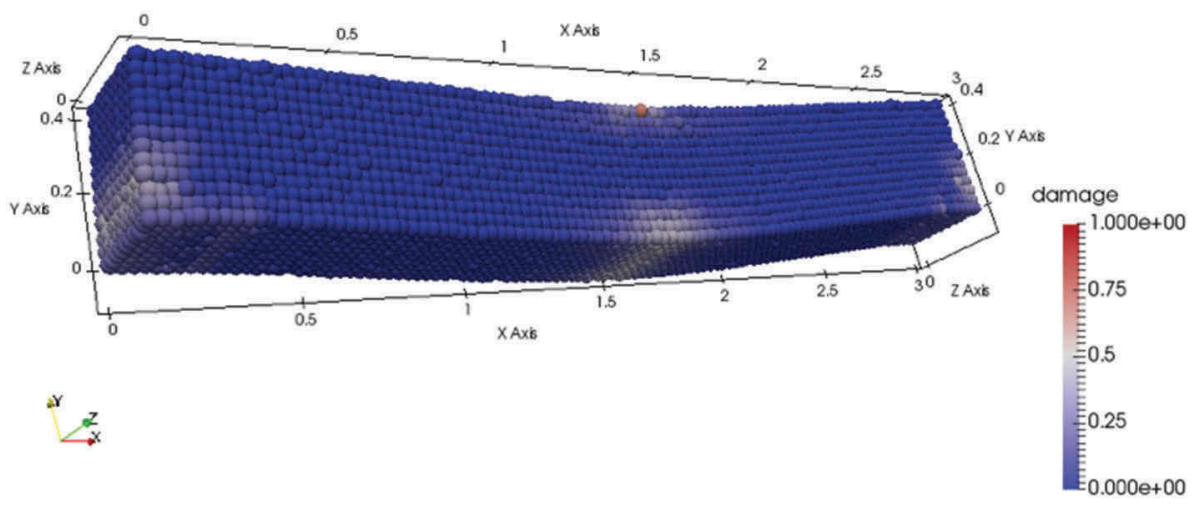

Figure 17. Damage for $\delta=0.37 \delta_{\max }$ (displacements scaled 10 times).

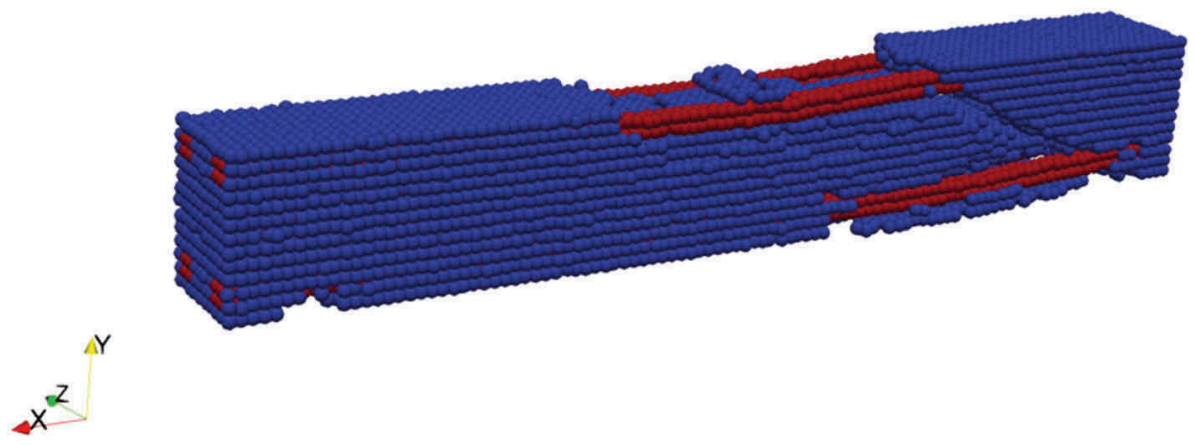

Figure 18. Perspective of the deformed configuration for $\delta=0.79 \delta_{\max }$ (totally damaged nodes were removed).

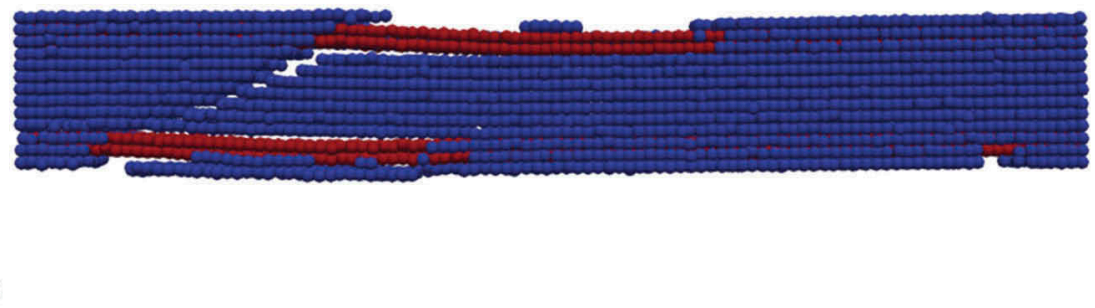

Figure 19. Deformed configuration for $\delta=0.79 \delta_{\max }$ (totally damaged nodes were removed).

test, a cubic specimen of concrete is compressed until failure using a calibrated compression machine. For the simulation, the prismatic volume represented in Figure 21 with dimensions $150 \times 150 \times 170 \mathrm{~mm}^{3}$ was considered. The volume is divided into three vertical layers represented in blue and grey. The middle layer in grey with dimensions $0.150 \mathrm{~m} \times 0.150 \mathrm{~m} \times 0.150 \mathrm{~m}$ corresponds to the test specimen in plain concrete of grade C60/75 with elastic modulus $E_{c}=39 \mathrm{GPa}$ and 


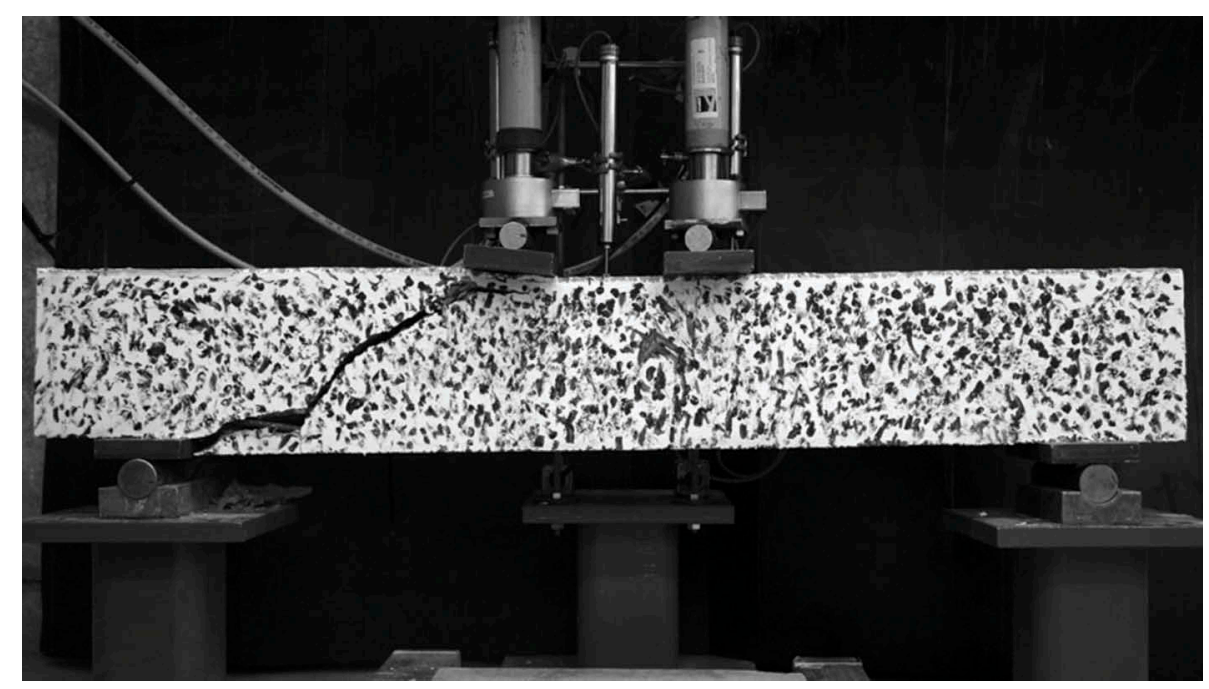

Figure 20. Typical mode of failure exhibited by beams without transverse reinforcement in laboratory experiments (photo courtesy Yuanzhang Yang).

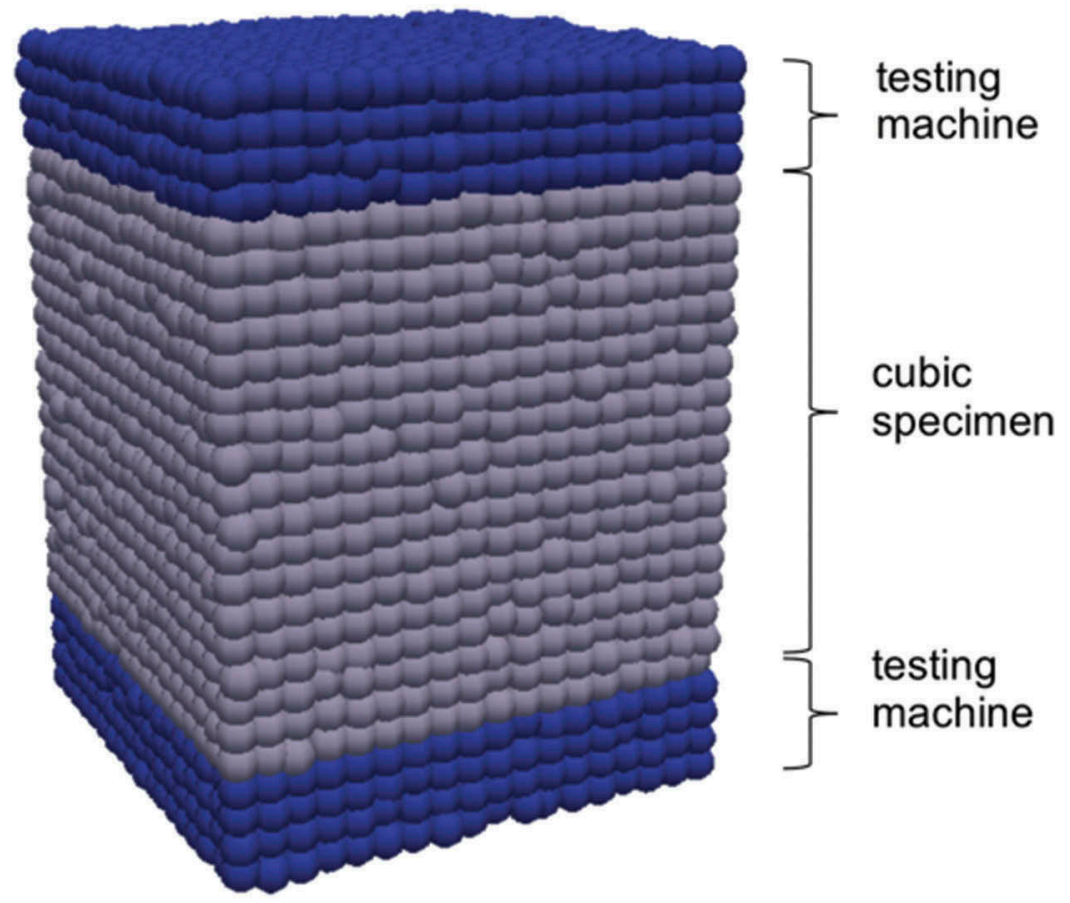

Figure 21. Particle model for simulating compressive strength (8425 particles and approximately 1.8 million bonds).

compressive strength $f_{c}=68 \mathrm{MPa}$ (CEN, 1992). The upper and lower layers in blue correspond to the plates of the compression machine. As a simple hypothesis, we consider the plates to be completely fixed to the 
specimen. The interface connections between particles of the specimen and particles of the machine are assumed to have the same properties as the specimen particles. Connections between particles of the upper and lower layers are considered to be one hundred times stiffer than the bonds corresponding to the specimen in order to simulate the effect of the testing machine. A uniformly distributed array of 8425 particles was considered initially. The particles in the top and bottom layers were constrained in the horizontal directions, and a compressive displacement of $\delta_{\max }=0.2 \mathrm{~mm}$ was applied in the vertical direction at a constant rate of $2.5 \mathrm{e}-3 \mathrm{~mm} / \mathrm{s}$.

The results of the simulation were obtained in terms of damage (as defined in Equation (34)) and the specimen's stress-strain response. The stress-strain response obtained from the model is compared to the Eurocode analytical curve for grade C60/70 concrete (CEN, 1992) in Figure 22.

For the numerical model, the peak stress was $72.9 \mathrm{MPa}$ at the strain of $\varepsilon_{c}=0.26 \%$, while according to the experimental benchmark (CEN, 1992) this value should be $68.0 \mathrm{MPa}$ at a strain of $0.25 \%$. Figure 23 shows a sequence of plots representing the damage levels at different strains, starting immediately before maximum resistance $\left(\varepsilon_{c}=0.26 \%\right)$ and afterwards during the brittle softening. Figure 24 shows the typical crack pattern obtained for this laboratorial experiment (Del Viso, Carmona, \& Ruiz, 2008).

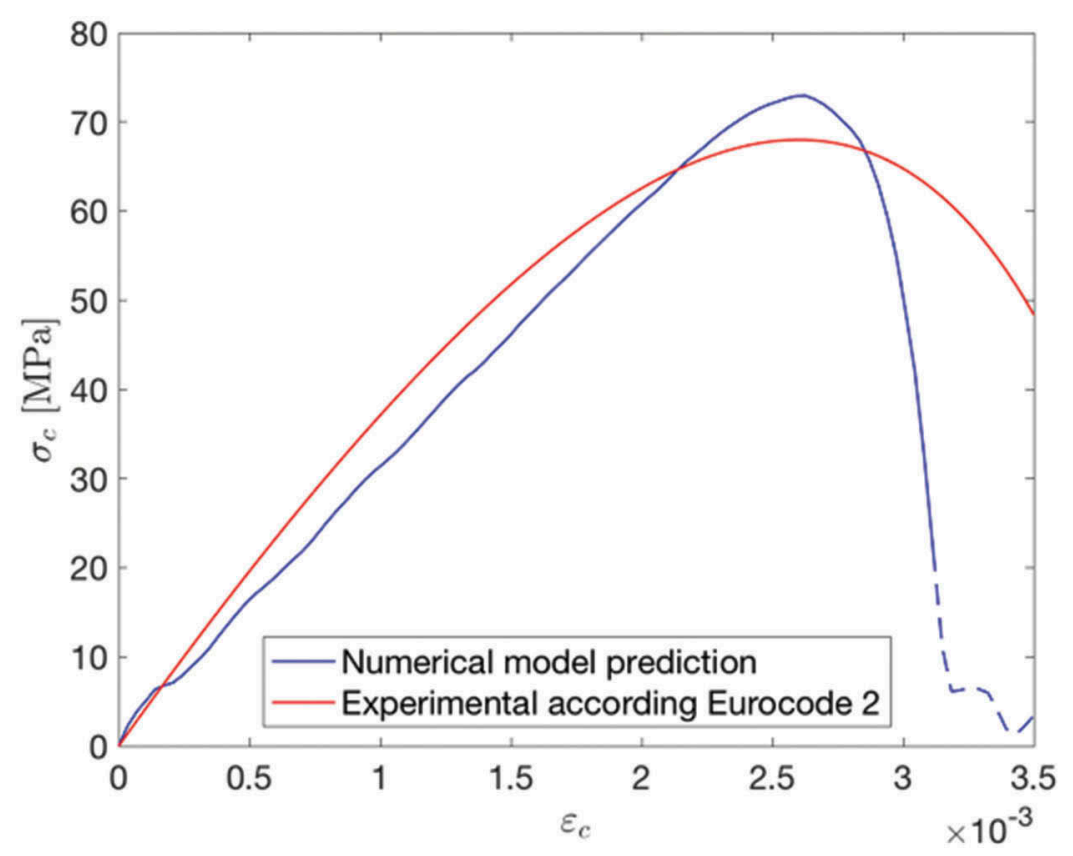

Figure 22. Comparison of stress-strain in compression from the numerical model and experiments (CEN, 1992). 

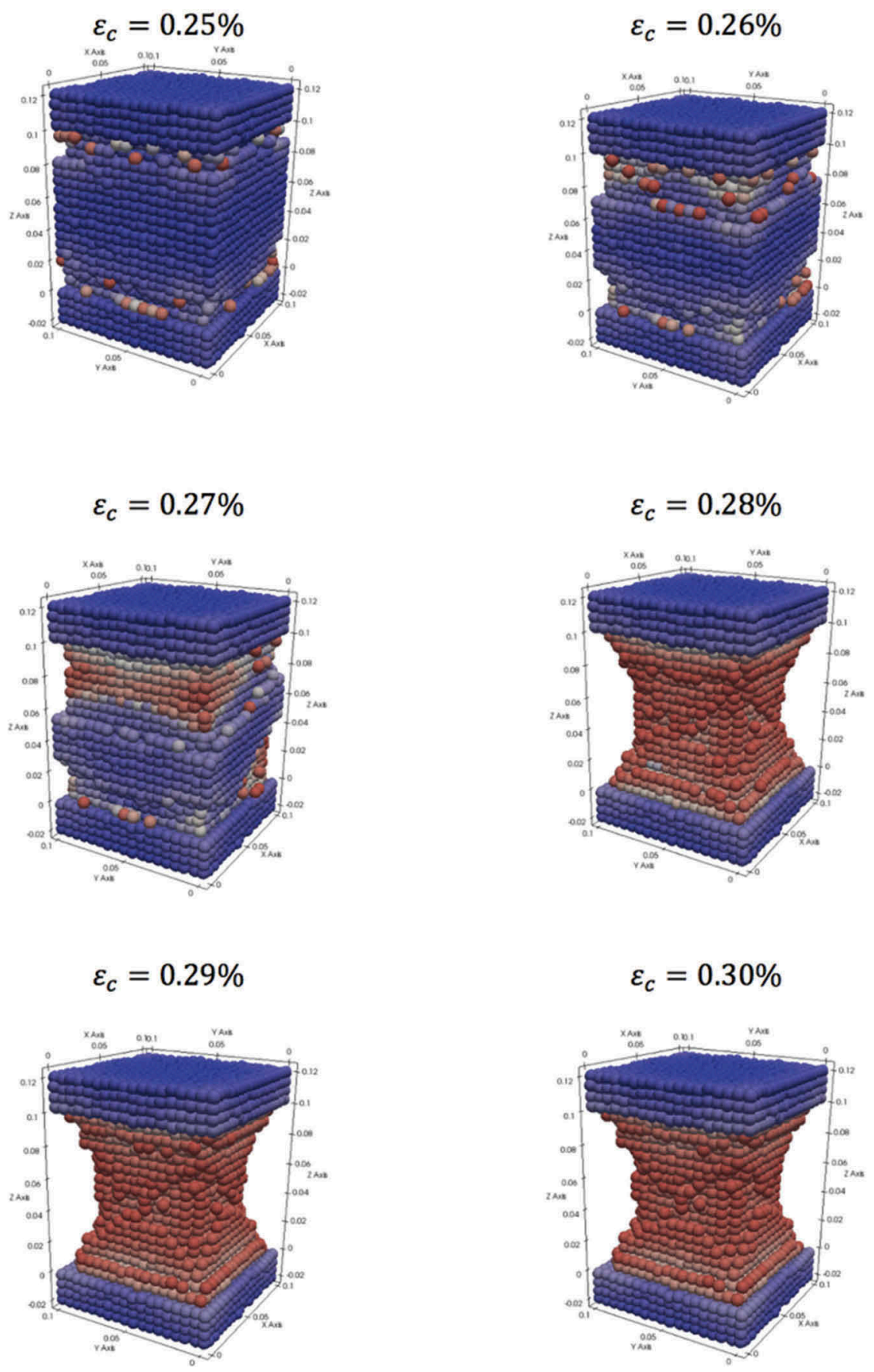

Damage

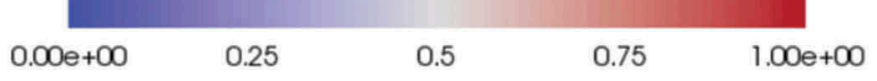

Figure 23. Damage evolution (pre-peak $\varepsilon_{c} \leq 0.26 \%$, fragile softening $\varepsilon_{c}>0.26 \%$ ). 


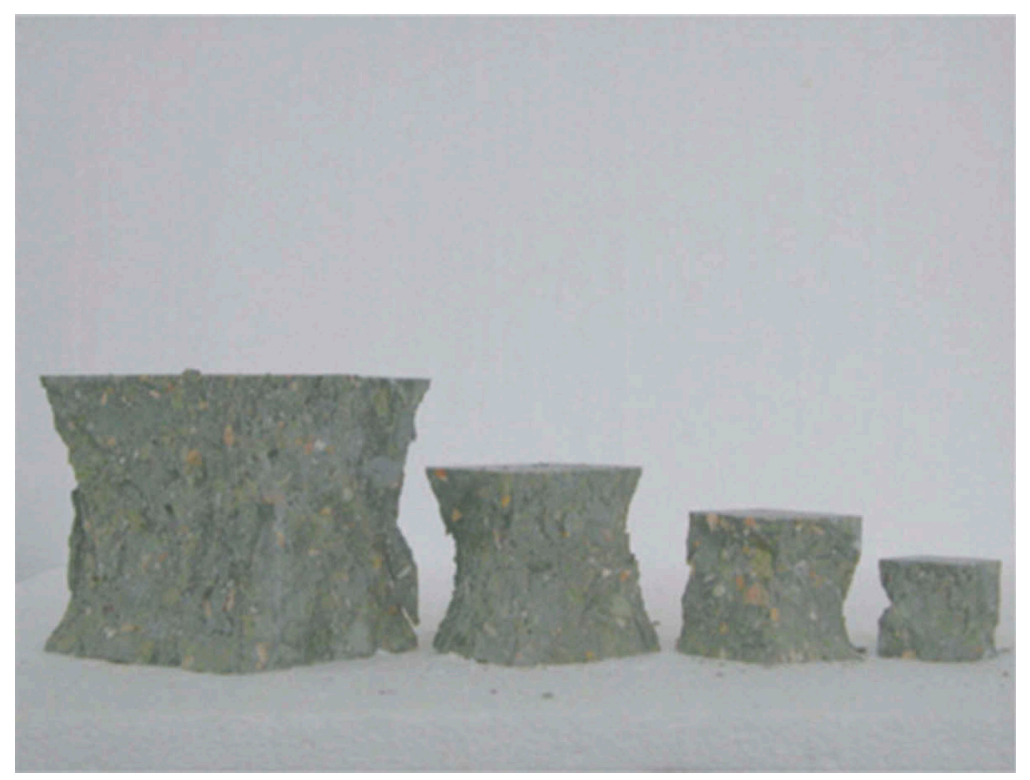

Figure 24. Crack patterns obtained in laboratorial experiments (Del Viso et al., 2008).

From the simulation, it is apparent that the damage onset begins at the boundaries, before a sudden brittle failure of the specimen occurs. The damage pattern from Figure 23 is similar to that obtained in laboratorial tests shown in Figure 24: all four exposed faces cracked approximately equally, with little damage to the faces in contact with the machine. Particles located near the free edges and free faces have less interactions (bonds) with other particles, when compared with particles located in the specimen's interior, for geometrical reasons. Therefore, the damage tends to initiate at the unconfined boundaries where fewer connections exist.

A certain degree of realism was obtained in replicating the cracking patterns of concrete; however, the experimental and simulation for the stress-strain relations are quite different. Before the peak strength, the numerical model behaviour is almost linear and does not able to resemble the slight and gradual reduction of stiffness that is shown in the experimental curve. Furthermore, the post-peak behaviour is brittle with a very abrupt reduction of strength, and not as soft as the one described in the experimental stress-strain curve. Concrete is a complex material with multiple scales; for instance, despite the fact that aggregates and cement paste are known to have linear elastic behaviour, the combination of both results in a non-linear material due to the interface transition zone between aggregates and the cement (see Scrivener et al., 2004). Therefore, the micro-brittle model used may be too simple to describe completely the complexity of concrete. Nevertheless, for the described 
examples the model has the advantage of predicting qualitatively the cracking and damage patterns without requiring any additional assumptions for the specific models.

\section{Conclusions}

A novel class of interaction functions are proposed for the computational implementation of peridynamic theory under explicit time integration schemes. The class of functions is very general since it consists of substituting the peridynamic stretch measure with a modified stretch making it faster to compute. This adaptation allows any constitutive model (linear elasticity, plasticity, or damage) to be redefined. The modified stretch was shown to converge to the stretch measure originally defined by Silling (Silling, 2000) when the strains are small. Furthermore, the equivalent stability conditions apply when using modified stretch under the explicit time integration schemes. We also presented a simple relation between stretch and modified stretch.

This new interaction function was implemented in a time explicit integration scheme. In the speed benchmark tests, gains of more than $11 \%$ were obtained in comparison with the traditional formulation. Good agreement was obtained for the validations of the method under linear elastic conditions against classical models. The capacity to reproduce cracking patterns and capacity of computation was demonstrated using three-dimensional examples: a simply supported steel reinforced concrete beam and a cube of plain concrete subjected to compression. Despite the limitations encountered due to the simplicity of the micro-brittle model employed, the presented examples demonstrate a certain capacity for reproducing crack and damage patterns. Therefore, the proposed strategy might in future also be applied using more refined models.

\section{Data access statement}

All data are provided in full in Sections 3 to 5 of this paper.

\section{Disclosure statement}

No potential conflict of interest was reported by the authors.

\section{Funding}

This research was supported by the Engineering and Physical Sciences Research Council (EPSRC) in the grant [EP/M020908/1] 'Concrete Modelled Using Random Elements'. 


\section{ORCID}

John Orr (D) http://orcid.org/0000-0003-2687-6353

\section{References}

Agwai, A., Guven, I., \& Madenci, E. (2011). Predicting crack propagation with peridynamics: A comparative study. International Journal of Fracture, 171(1), 65.

Besson, J. (2010). Continuum models of ductile fracture: A review. International Journal of Damage Mechanics, 19(1), 3-52.

CEN. (1992). EN 1992-1 Eurocode 2: Design of concrete structures. Brussels, Belgium: European Committee for Standardization.

Chaboche, J. (1978). Description thermodynamique et phénoménologique de la viscoplasticité cyclique avec endommagement (Publication No. 1978-3). 92320 Chatillon. France: Office National dEtudes et de Recherches Aérospatiales.

Day, A. (1965). An introduction to dynamic relaxation (Dynamic relaxation method for structural analysis, using computer to calculate internal forces following development from initially unloaded state). The Engineer, 219, 218-221.

de Souza Neto, E. A., Peric, D., \& Owen, D. R. (2011). Computational methods for plasticity: Theory and applications. New Jersey, USA: John Wiley \& Sons.

Del Viso, J., Carmona, J., \& Ruiz, G. (2008). Shape and size effects on the compressive strength of high-strength concrete. Cement and Concrete Research, 38(3), 386-395.

Emmrich, E., \& Weckner, O. (2007). The peridynamic equation and its spatial discretisation. Mathematical Modelling and Analysis, 12(1), 17-27.

Gere, J. M., \& Goodno, B. J. (2012). Mechanics of materials, R.E. Services, ed. Atlanta, Georgia, USA: Global Engineering: Christopher M. Shortt.

Gerstle, W. (2015). Introduction to practical peridynamics: Computational solid mechanics without stress and strain. Singapore, Singapore: World Scientific.

Gerstle, W., Sau, N., \& Silling, S. (2007). Peridynamic modeling of concrete structures. Nuclear Engineering and Design, 237(12), 1250-1258.

Hibbitt, Karlsson \& Sorensen, Inc. (1998). ABAQUS/standard: User's manual. Providence, RI: Hibbitt, Karlsson \& Sorensen.

Higham, D. J., \& Higham, N. J. (2005). MATLAB guide. Philadelphia, USA: Siam.

Hu, W., Ha, Y. D., \& Bobaru, F. (2011). Modeling dynamic fracture and damage in a fiber-reinforced composite lamina with peridynamics. International Journal for Multiscale Computational Engineering, 9(6), 707-726.

Jason, L., Huerta, A., Pijaudier-Cabot, G., \& Ghavamian, S. (2006). An elastic plastic damage formulation for concrete: Application to elementary tests and comparison with an isotropic damage model. Computer Methods in Applied Mechanics and Engineering, 195(52), 7077-7092.

Jing, L. (2003). A review of techniques, advances and outstanding issues in numerical modelling for rock mechanics and rock engineering. International Journal of Rock Mechanics and Mining Sciences, 40(3), 283-353.

Kachanov, L. M. (1958). Time of the rupture under creep conditions. Известия Академии Наук СССР Отделение Тескхнических Наук, 8, 26-31.

Kilic, B. (2008). Peridynamic theory for progressive failure prediction in homogeneous and heterogeneous materials. Tucson, USA: The University of Arizona.

Kilic, B., \& Madenci, E. (2010). Coupling of peridynamic theory and the finite element method. Journal of Mechanics of Materials and Structures, 5(5), 707-733. 
Le, Q., \& Bobaru, F. (2018). Surface corrections for peridynamic models in elasticity and fracture. Computational Mechanics, 61(4), 499-518.

Lemaitre, J. (1985). A continuous damage mechanics model for ductile fracture. Journal of Engineering Materials and Technology, 107(1), 83-89.

Macek, R. W., \& Silling, S. A. (2007). Peridynamics via finite element analysis. Finite Elements in Analysis and Design, 43(15), 1169-1178.

Miranda, H. D., Williams, C. J. K., \& Orr, J. (2016). An explicit method for simulation of quasi-brittle materials and structures based on peridynamic theory, in 12th World Congress on Computational Mechanics. South Korea: Seoul.

Murakami, S. (2012). Continuum damage mechanics: A continuum mechanics approach to the analysis of damage and fracture (Vol. 185). Berlin, Germany: Springer Science \& Business Media.

Otter, J. (1965). Computations for prestressed concrete reactor pressure vessels using dynamic relaxation. Nuclear Structural Engineering, 1(1), 61-75.

Scrivener, K. L., Crumbie, A. K., \& Laugesen, P. (2004). The interfacial transition zone (ITZ) between cement paste and aggregate in concrete. Interface Science, 12(4), 411-421.

Silling, S. A. (2000). Reformulation of elasticity theory for discontinuities and long-range forces. Journal of the Mechanics and Physics of Solids, 48(1), 175-209.

Silling, S. A., Epton, M., Weckner, O., Xu, J., \& Askari, E. (2007). Peridynamic states and constitutive modeling. Journal of Elasticity, 88(2), 151-184.

Silling, S. A., \& Askari, E. (2005). A meshfree method based on the peridynamic model of solid mechanics. Computers \& Structures, 83(17), 1526-1535.

Squillacote, A. H., \& Ahrens, J. (2007). The paraview guide (Vol. 366). New York, USA: Kitware.

Wu, J. Y., Li, J., \& Faria, R. (2006). An energy release rate-based plastic-damage model for concrete. International Journal of Solids and Structures, 43(3-4), 583-612.

$\mathrm{Xu}, \mathrm{H}$., \& Arson, C. (2014). Anisotropic damage models for geomaterials: Theoretical and numerical challenges. International Journal of Computational Methods, 11(02), 1342007.

Yu, T., Teng, J., Wong, Y., \& Dong, S. (2010). Finite element modeling of confined concrete-I: Drucker-Prager type plasticity model. Engineering Structures, 32(3), $665-679$.

\section{Appendix A. Derivation of the micromodulus}

In this appendix, we derive the micromodulus, and show that it is the same using the modified bond stretch $\bar{s}$ or the bond stretch $s$ in the definition of $f$. The tensor-valued function called micromodulus is defined by

$$
\mathbf{C}(\boldsymbol{\xi})=\frac{\partial \boldsymbol{f}}{\partial \boldsymbol{\eta}}(\mathbf{0}, \boldsymbol{\xi}) \quad \forall \boldsymbol{\xi} .
$$

Considering

$$
\boldsymbol{f}(\mathbf{\eta}, \boldsymbol{\xi})=\frac{\boldsymbol{\eta}+\boldsymbol{\xi}}{|\boldsymbol{\eta}+\boldsymbol{\xi}|} c s
$$

and substituting in Equation (A1) we obtain

$$
\mathbf{C}(\boldsymbol{\xi})=\frac{\partial f}{\partial \boldsymbol{\eta}}(\boldsymbol{\eta}, \boldsymbol{\xi}) \quad \mid \boldsymbol{\eta}=\mathbf{0},
$$


which in indicial notation is equivalent to

$$
C_{i j}=\frac{\partial}{\partial \eta_{j}}\left(\frac{\eta_{i}+\xi_{i}}{\left(\eta_{k}+\xi_{k}\right)^{1 / 2}} c s\right) \quad \mid \eta_{i}=0 .
$$

Developing Equation (A4) leads to

$$
C_{i j}=\frac{\partial}{\partial \eta_{j}}\left(\frac{\eta_{i}+\xi_{i}}{\left(\eta_{k}+\xi_{k}\right)^{1 / 2}}\right) c s+\frac{\eta_{i}+\xi_{i}}{\left(\eta_{k}+\xi_{k}\right)^{1 / 2}} c \frac{\partial s}{\partial \eta_{j}} \quad \mid \eta_{i}=0
$$

For $\boldsymbol{\eta}=0$, the first term of Equation (A5) vanishes, resulting in the following expression:

$$
C_{i j}=\frac{\xi_{i}}{\left(\eta_{k}+\xi_{k}\right)^{1 / 2}} c \frac{\partial s}{\partial \eta_{j}} \quad \mid \eta_{i}=0
$$

Considering Equations (20) and (21), it is possible to conclude that $\frac{\partial s}{\partial \eta_{j}}=\frac{\partial \bar{s}}{\partial \eta_{j}}$ when $\boldsymbol{\eta}=\mathbf{0}$. Therefore, substituting this derivative given in Equation (20) or Equation (21) with $\boldsymbol{\eta}=\mathbf{0}$, in Equation (A6), leads to the micromodulus defined by

$$
C_{i j}=\frac{\xi_{i}}{\left(\eta_{k}+\xi_{k}\right)^{1 / 2}} c \frac{\xi_{j}}{\left(\eta_{k}+\xi_{k}\right)^{2}}=c \frac{\xi_{i} \xi_{j}}{\left(\eta_{k}+\xi_{k}\right)^{3 / 2}} \quad \text { or } \quad \mathbf{C}=\mathrm{c} \frac{\xi \otimes \xi}{|\xi|^{3}}
$$

